\title{
Automation System for Transporting Highly Radioactive Samples of the Irradiation Facility of the IBR-2 Reactor
}

\author{
M. V. Bulavin ${ }^{a,}$ *, P. A. Dorofeev ${ }^{a}$, A. V. Galushko ${ }^{a}$, and A. V. Altynov ${ }^{a}$ \\ a Joint Institute for Nuclear Research, Moscow oblast, Dubna, 141980 Russia \\ *e-mail: bulavin85@inbox.ru
}

Received September 23, 2020; revised October 28, 2020; accepted October 29, 2020

\begin{abstract}
This paper presents the results of an automation of the processes of measuring the dose rate and movement of highly active samples in the controlled zone of the third beam of the IBR-2 reactor. A detailed analysis of the choice of an automated system based on a robotic manipulator with a video-surveillance system and distance measurement, as well as a dosimetric complex for determining the dose rate in the place of its operation, has been carried out. The processes of constructing algorithms and programs to control the robot are described in detail. It has been established that the system operates uninterruptedly and reliably in the fields of high ionizing radiation; is capable of almost completely replacing a person when performing such a work; and, most importantly, significantly_up to $27.4 \%$-reduces the annual dose received by personnel during operation.
\end{abstract}

Keywords: robotic manipulator, automation, ionizing radiation, nuclear materials and radioactive substances, irradiation, nuclear research facility

DOI: $10.1134 /$ S1547477121020059

\section{INTRODUCTION}

The facility for irradiating various materials at a modernized IBR-2 reactor with the purpose of studying their radiation resistance has been in operation since 2012 [1-4]. The high density of the neutron flux interacting with the sample under investigation leads to a quite high induced activity of both the sample itself and the structural elements of the irradiation facility. As a rule, all samples after irradiation can be conditionally divided into two groups: those requiring the urgent neutron activation analysis of short-lived isotopes (from a few hours to 2 days after irradiation), for example, [5-6], and those that do not require such an analysis [7-11]. Taking into account the fact that, in the first hours after irradiation, the main contribution to the induced radioactivity is usually made by short-lived isotopes; then the transportation of their samples from the structural elements of the facility may become a problem because of extremely high values of radioactivity (up to $1 \mathrm{~Sv} / \mathrm{h}$ ).

This problem is aggravated by the fact that, in the reactor-controlled zone $(\mathrm{CZ})$, where the irradiation facility operates, the project does not provide for any automated transport or pneumatic transport systems and the transportation of samples is executed either manually or by means of mechanical gripping devices of various lengths. It is not always convenient or possible to use such gripping devices to take off highly active samples, mainly because of a potentially high radioac- tive loading on the personnel related to a long term presence near the sample. This is why carrying out important and interesting experiments on irradiation at the facility either involves a high risk that personnel will receive high dosages that exceed the maximum permissible dose established by ionizing radiation regulations or is completely forbidden.

This problem can be solved, for example, in the reactor zone by using a system based on a robotic manipulator with a grip which is capable of transporting highly radioactive samples from structural elements of the irradiation facility to the storage or the transport container inside the controlled reactor zone of the IBR-2 reactor with the possibility of simultaneous control over the irradiation dose rate during operation.

Therefore, the aim of this study is to create such a system and test its working capacity in the fields of high ionizing radiation near highly radioactive samples during their transportation.

To achieve this aim, it is necessary to solve the following problems:

(1) carry out installation and startup-setup work on the automated manipulator, video-surveillance system, and dosimetric control in the reactor $\mathrm{CZ}$ when working at the irradiation facility of the IBR-2 reactor;

(2) use the software of the robotic manipulator to create an algorithm for measuring the dose rate at various points of the $\mathrm{CZ}$ and transporting containers with 


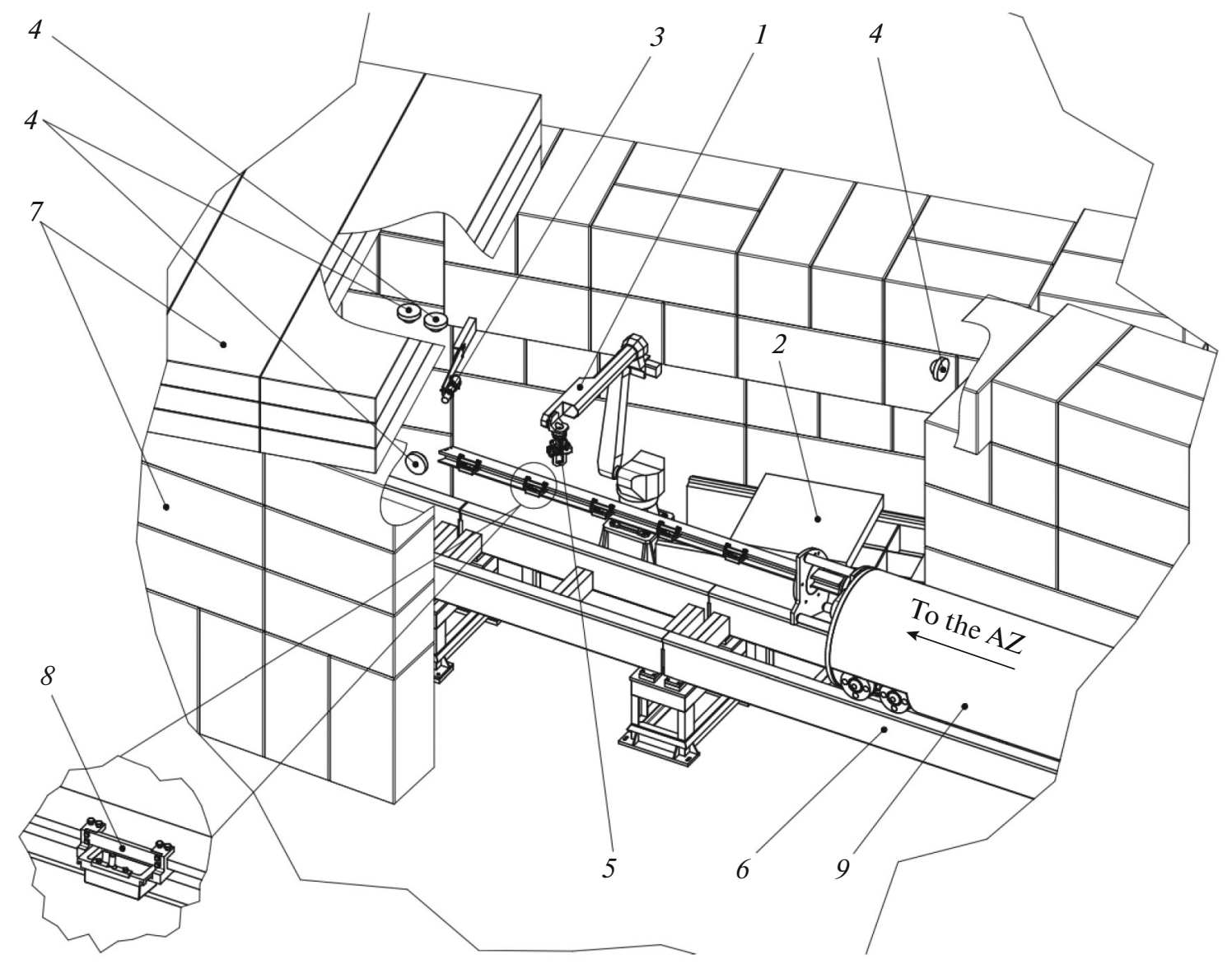

Fig. 1. Automated system of the robotic manipulator in the controlled zone of the IBR-2: (1) robot, (2) SRW storage, (3) detection unit of the ionizing gamma-radiation sensor, (4) high-resolution cameras of the video-surveillance system, (5) pneumatic gripper with a laser target designator and a high-resolution camera, (6) rail path, (7) biological shielding of the CZ, (8) containers for samples arranged at the transport double tee $(L=3 \mathrm{~m})$ of the irradiation facility, and (9) irradiation facility; AZ is the active zone with the VZ-303 water moderator of the IBR-2 reactor.

radioactive samples to various places of the irradiation facility or to the solid radioactive waste (SRW) storage;

(3) in the course of carrying out reactor cycles, irradiate samples and measure the dose rate at different points of the $\mathrm{CZ}$, as well as transport containers with samples after irradiation according to the created algorithms;

(4) make conclusions and draw an inference on the working capacity of such a system in the high ionizing radiation fields and on a real reduction of the radiation-absorbed dose received by personnel.

\section{PRINCIPLE OF OPEARATION OF THE AUTOMATED SYSTEM OF THE MANIPULATOR \\ WITH A VIDEO-SURVEILLANCE SYSTEM AND DOSIMENTRIC CONTROL}

Figures 1 and 2 show the automated system of robotic manipulator. The robotic manipulator, man- ufactured by FANUC (FANUC Europe Corporation S.A.) (1), is located to the right of the rail track (6) along with the irradiation facility (9) with samples in transport containers $(8)$ moves towards the active zone or away from it.

The robot is equipped with a pneumatic gripper (5) with a laser target designator (10) (Fig. 2) for automatic gripping and transportation of radioactive wastes, as well as a high-resolution camera (11) for remote control. Remote programming and control over the robotic manipulator are also performed by means of video cameras (4) arranged at different points of the $\mathrm{CZ}$, overlapping all fields of vision. Among them, there are 3 Active Cam AC-D6124 rotating IP-video cameras with a possibility of $25 \times$ optical zoom and 2 static Trassir TR-D8121WDIR-2 high-resolution cameras with a frequency higher than 25 frames per second and with a $32 \times$ zoom. One of the static cameras is arranged at the pneumatic gripper; 


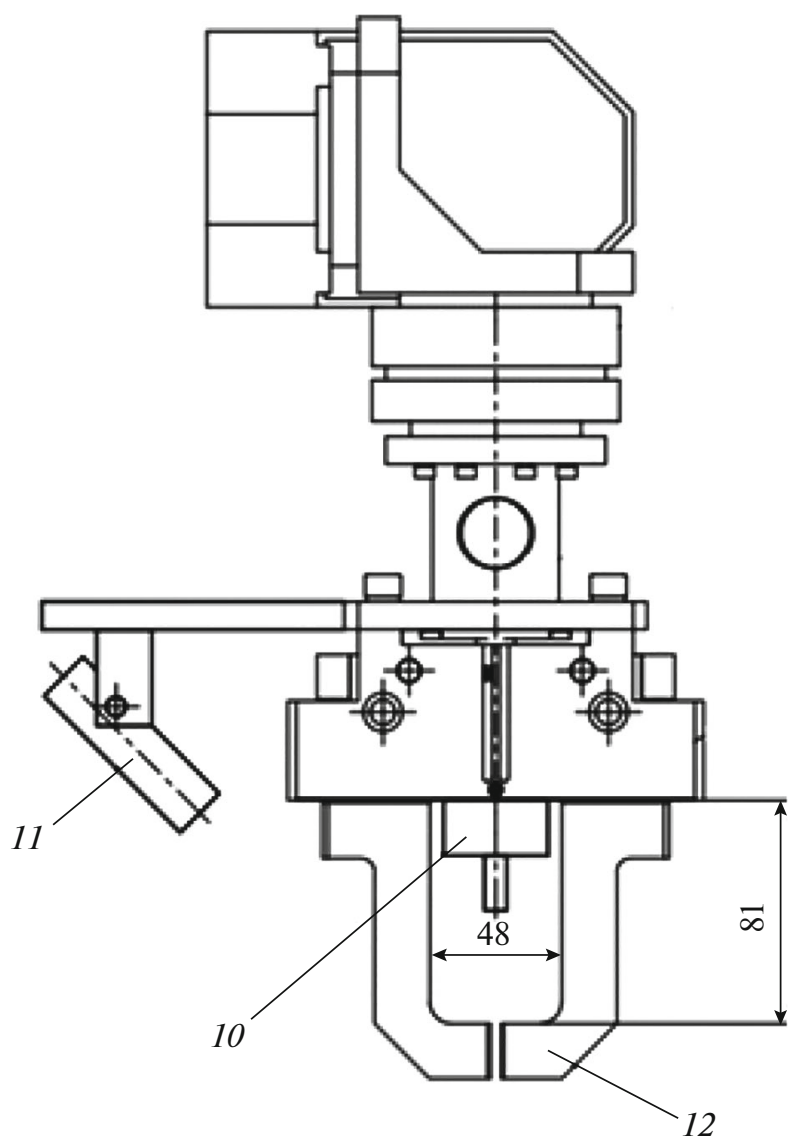

Fig. 2. Pneumatic gripper of the robot: (10) laser target designator, (11) high-resolution video-surveillance camera, and (12) movable jaws.

another, under the biological shielding (7), is at the closest distance from the AZ.

Irradiated highly radioactive containers with samples $(8)$ are transported from the transport double tee to a special SRW storage (2), which has multiple storage cells. The dosimetric system with a gamma radiation (3) detection unit is arranged under the biological shielding of the $\mathrm{CZ}$ in the immediate vicinity of the pneumatic gripper of the robot.

The principle of operation of the automated system is as follows. After finishing a standard reactor cycle, the irradiation facility is transported to the $\mathrm{CZ}$ to the required point at the rail track, which is related to the coordinate system of the robot. The location of the facility at a given point is fixed by the video surveillance system. At the first stage, before gripping the container holding the samples, it is necessary, from afar, to estimate the radiation background near the facility. To do this, by means of a control console (CC) of the robot (Fig. 3), a previously written program algorithm of its operation is started up; following this algorithm, the pneumatic gripper moves to the detection block (3), which is connected by a $25-\mathrm{m}$ cable with the measurement panel located at a distance from the CZ. The controller of the robot control is located in a safe zone at a distance of $\sim 30 \mathrm{~m}$ from the $\mathrm{CZ}$ and is connected with the $\mathrm{CC}$ by means of a long cable, which enables the operator to work far from where highly radioactive samples are located. The information of the dose rate is visualized on the display of the measurement panel and can be also visualized on the PC monitor.

After gripping the detection unit, the pneumatic gripper of the robot moves to a distance of $\sim 100 \mathrm{~mm}$ (measurement near the container) from the container of the irradiation facility which is planned to be removed and measures the dose rate for $30 \mathrm{~s}$ (Fig. 4); then it moves to a distance of $\sim 500 \mathrm{~mm}$ (the distance to a person with consideration for their arms outstretched during the working process) and measures the dose rate as well. After that, it returns the detection unit to its place and reverts to its off-duty position.
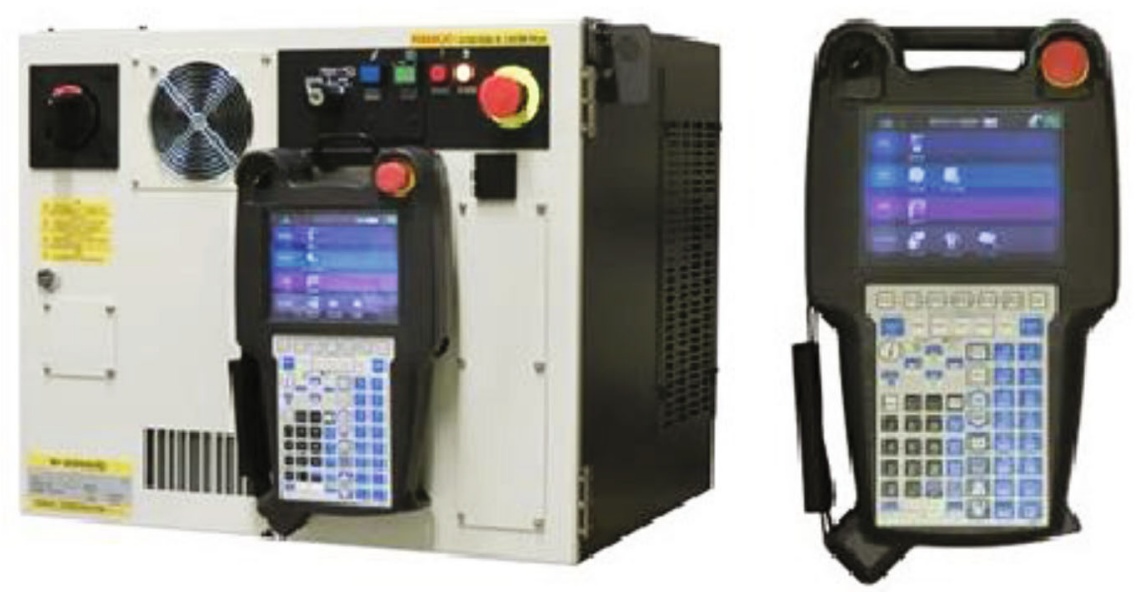

Fig. 3. Controller of robot operation and the control console. 


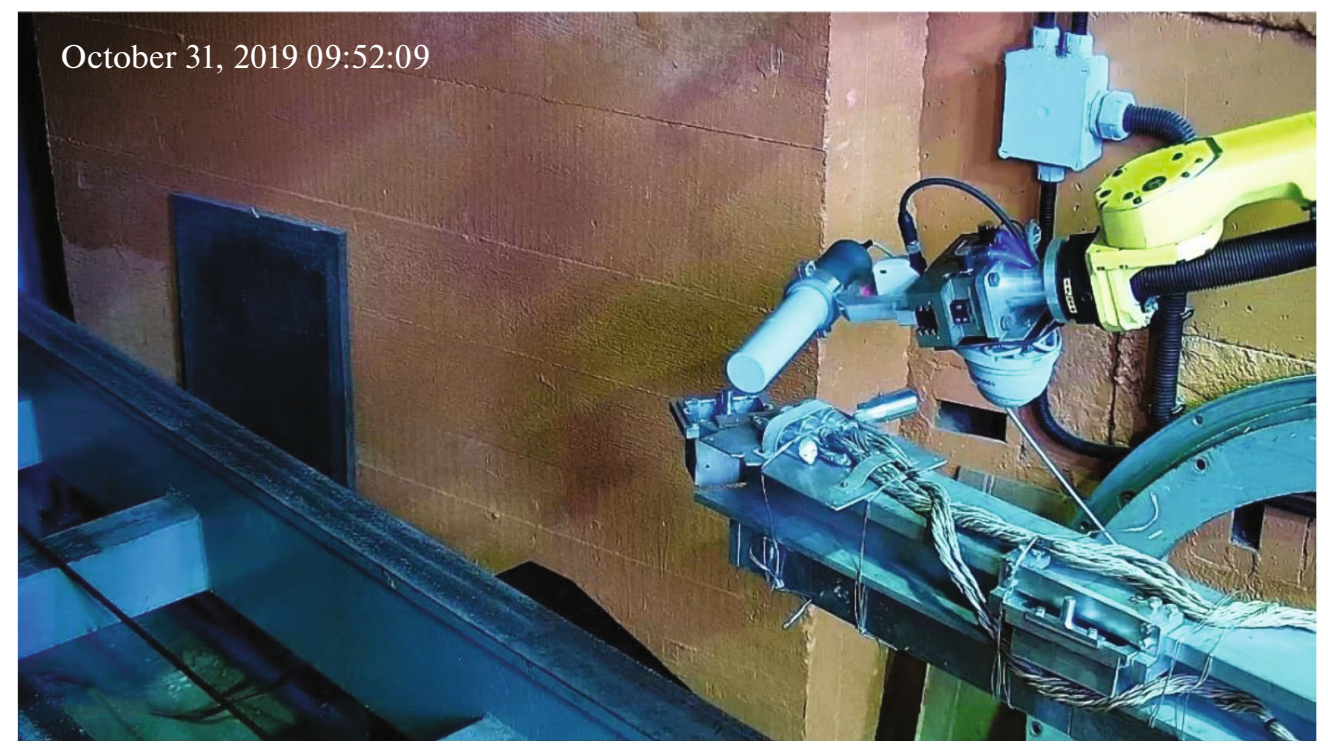

Fig. 4. Image of one of the video-surveillance cameras taken at the moment it measured the dose rate near the sample surface.

The data are stored and analyzed; after that, the operator starts the procedure of transporting the container(s) with samples to the SRW storage (Figs. 5a, 5b). For that, the next program algorithm starts to be applied; this algorithm is responsible for transporting one or several containers, depending on the needs of the experimenters. In the end of any program algorithm, the robot returns to its off-duty position; the irradiation facility is transported to the reactor hall up to the next irradiation cycle. The samples put into the container are stored there until it will be necessary to transport them for further investigations or utilization.

\section{CREATING ALGORITHMS FOR MEASURING THE DOSE RATE AND TRANSPORTING CONTAINERS WITH SAMPLES IN THE SOFTWARE OF THE ROBOTIC MANIPULATOR}

The operator uses the KAREL programming language to create the algorithms for controlling the FANUC robot manipulator. The KAREL language makes it possible to process data, connect to the corresponding equipment and control it, and communicate with the operator. KAREL is a mixture of highlevel languages such as PASCAL and PL/1 and includes the structures common for the high-level languages, as well as the functions designed especially for robotics.

The operation regime of the robotic manipulator in the process of measuring the dose rate and transporting containers is automatic and uses a previously set program algorithm. The "manual" control of the robot is used only in extreme cases, when the automatic operation regime is impossible for some reason.
The robot programming, first and foremost, consists of setting the main points and ways of motion between them in a given coordinate system. The coordinate system may be related directly to the robot axes, its position, the instrument (in this case, a pneumatic gripper), or the operation field (the irradiation facility).

To work with containers, the following coordinate system related to the irradiation facility was used: the $\mathrm{X}$ and $\mathrm{Y}$ coordinates are in the plane of the profile of the double tee for sample installation and are directed along its length and width, while the $\mathrm{Z}$ coordinate is normal to its surface.

Since the value of displacement in the process of fixing the containers with samples at the transport double tee is larger than the error of axial displacements of the robot, it was impossible to perform unloading by a cycle of the same manipulations with a displacement along the $x$ axis (the Offset function); therefore, a special program block was created for transportation of each container.

The manipulator is programmed through the remote control console (CC). Using the commands of motion on the $\mathrm{CC}$, the operator drives the robot to the required position and writes down the coordinates of this point into the program, as well as the velocity of motion and the type of interpolation (smoothing the trajectory when passing through the point, if it is not the ultimate point in the written robot motion). After that, the operator drives the robot to the next position and saves the next point in the program. This procedure continues until the robot arrives at the required point along a safe trajectory-in this case, to the point where the container is gripped. 
(a)

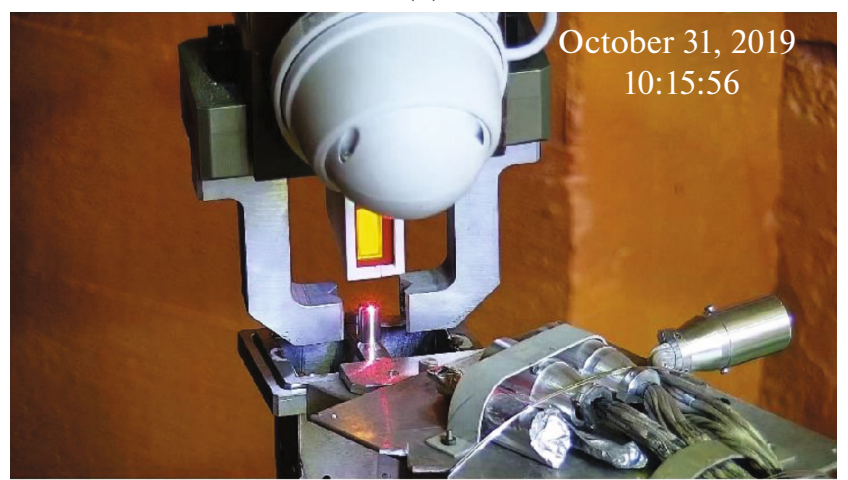

(b)

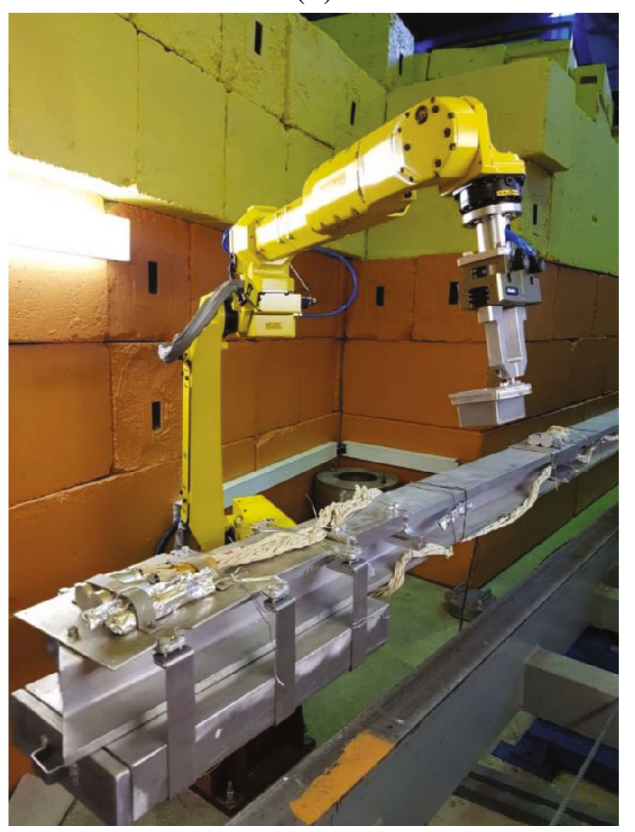

Fig. 5. Robot in the process of container transportation: (a) before gripping and (b) in the process of transportation to the SRW storage.

Next, the call to the subroutine of container gripping by jaws occurs. After it is completed, the operator writes down the points of the trajectory of container transportation to the SRW storage, where the call to the subroutine of unclasping the movable jaws of the pneumatic gripper occurs. After that, the operator draws the robot to the point of approach, if it is necessary to take off other containers, or returns to its offduty position, if the sample unloading is finished.

When working out the algorithm for container transportation, it is necessary to consider one important peculiarity. After transporting the irradiation facility to the $\mathrm{CZ}$, the robot is closed in the bounded space between the biological shielding wall and the facility itself; the containers with samples are fixed at the double tee at a distance of about $1.5 \mathrm{~m}$ from the point of robot installation. Therefore, the trajectory of the manipulator from its parking place and the SRW storage to the place where the containers are fixed is complicated and long in order to exclude the possibility of collisions between the robot elements and constructions surrounding it.

Some examples of algorithms for measuring the dose rate and container transportation are shown in Figs. 6a and 6b.

\section{USE OF THE ATOMATED SYSTEM FOR SAMPLE TRANSPORTATION AND MEASURING THE DOSE RATE UNDER THE CONDITIONS OF REAL REACTOR EXPERIMENT}

Exploitation of the automated system under conditions of a real reactor experiment started in November 2018. In this time, the automated system was used in ten cycles of IBR-2 reactor operation; several tens of measurements of the dose rate and container motions were performed. All containers with samples were located at different places of the transport double tee 100 to $3000 \mathrm{~mm}$ from the surface of the reactor water moderator and had different values of induced radioactivity after irradiation, namely, from 10 to $800 \mathrm{mSv} / \mathrm{h}$. The electronics of the automated system did not experience any failures throughout the entire operating period.

Table 1 shows the values of the average dose rate of gamma radiation for a year near the containers with samples (at distances of 100 and $500 \mathrm{~mm}$ ) subjected to irradiation near the reactor which had to be transported, according to the conditions of the experiment. The measurement results obtained in the experiment were used to calculate the annual dose that would be received by the personnel of the irradiation facility when transporting containers manually or using mechanic grippers, as well as by the radiation supervisor on duty that performs the measurements before the personnel starts working in the CZ. Experience has shown that the average time of operation in the $\mathrm{CZ}$ is $60 \mathrm{~s}$ for the radiation supervisor on duty and $180 \mathrm{~s}$ for the personnel of the irradiation facility for one cycle of reactor operation. For convenience's sake, the time of operation in Table 1 is presented in hours.

The following formula was used to calculate the average annual dose:

$$
A=D_{2} t_{2},
$$

where $D_{2}$ is the average annual dose rate from the container with samples at a distance of $50 \mathrm{~cm}$ from its surface, $\mathrm{mSv} / \mathrm{h} ; t_{2}$ is the average time of operation of the personnel in the $\mathrm{CZ}$ when measuring the dose rate and transporting the samples for 1 year, $h$.

The results of measurements and the calculations based on them have shown that the personnel performing short-term works on measuring the dose rate near the surface of the containers and their transpor- 
(a)

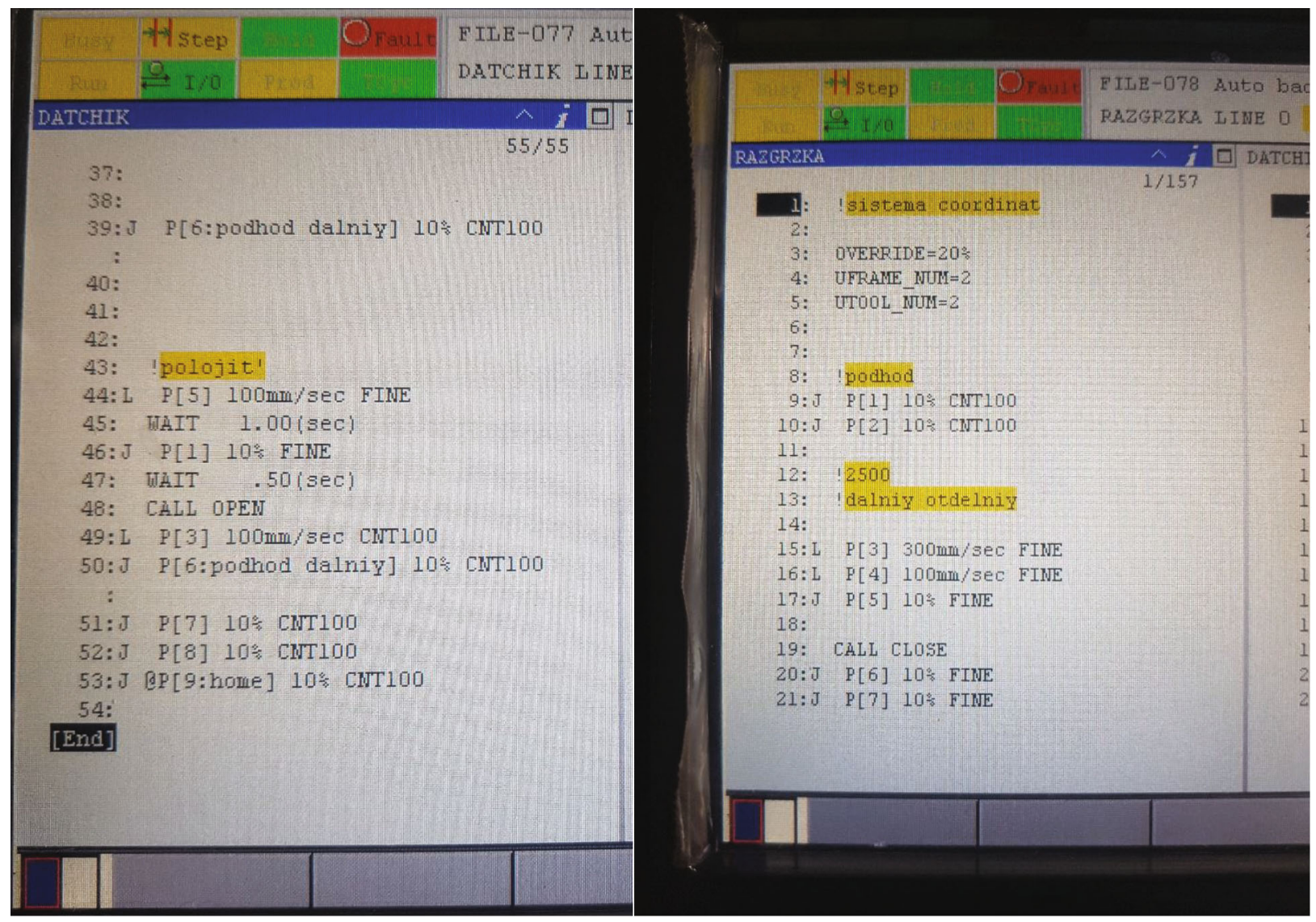

Fig. 6. Screenshots of algorithms from the control panel of manipulator: (a) algorithm for measuring the dose rate and (b) algorithm for transporting containers with highly radioactive samples.

tation receives an average annual dose from $\sim 9.1$ to $27.4 \%$ of the annual dose for the personnel of group A established in [14].

Such percentages are quite significant, since for neither the personnel operating as radiation supervi- sors on duty, nor for the personnel directly carrying out works at the irradiation facility, is this the only kind of activity related to operation with radioactive materials or substances. Therefore, without using this automated system, which would help in completely

Table 1. Average dose rate received by the personnel calculated based on the data obtained using the robotic manipulator

\begin{tabular}{l|c|c}
\hline & $\begin{array}{c}\text { Radiation } \\
\text { supervisor on duty }\end{array}$ & $\begin{array}{c}\text { Personnel of the irradiation } \\
\text { facility }\end{array}$ \\
\hline $\begin{array}{l}\text { Average annual dose rate } D_{1} \text { from the container with samples } \\
\text { at a distance of } 10 \mathrm{~cm} \text { from its surface, } \mathrm{mSv} / \mathrm{h}\end{array}$ & 273.13 & 273.13 \\
$\begin{array}{l}\text { Average annual dose rate } D_{2} \text { from the container with samples } \\
\text { at a distance of } 50 \mathrm{~cm} \text { from its surface, } \mathrm{mSv} / \mathrm{h}\left(\mathrm{M}_{50}\right)\end{array}$ & 10.93 \\
$\begin{array}{l}\text { Average time } t_{1} \text { of personnel operation in the } \mathrm{CZ} \text { when measuring } \\
\text { the dose rate and transporting the samples for } 1 \mathrm{cycle}\end{array}$ & 0.0167 \\
$\begin{array}{l}\text { of reactor operation, } \mathrm{h} \\
\begin{array}{l}\text { Average time } t_{2} \text { of personnel operation in the } \mathrm{CZ} \text { when measuring the } \\
\text { dose rate and transporting the samples for } 1 \text { year, h }\end{array}\end{array}$ & 0.05 \\
$\begin{array}{l}\text { Average annual dose } A, \mathrm{mSv} \\
\text { Part of the average annual dose for the personnel of group } A, \%\end{array}$ & 0.167 & 0.5 \\
\hline
\end{tabular}


replacing personnel, it is impossible for them to avoid an augmentation of the received annular dose. Since taking care of human health and labor safety by reducing the received annual dose is a foreground task for any organization that operates with radioactive materials or substances, creating such systems at research reactors or other nuclear-research facilities is very important and necessary.

\section{CONCLUSIONS}

The automation of the system of transporting highly radioactive samples and measuring the dose rate in the controlled zone of the 3rd beam of the IBR-2 reactor has made it possible to optimize the operation of personnel working at the irradiation facility. The result of this optimization is a significant-by nearly $30 \%$-reduction in the radiation-absorbed dose for the personnel. In addition, it makes it possible to transport samples with an extremely high radioactivity level immediately after the end of their irradiation, which was impossible before its creation. Since the reliability of this system operating in fields of high ionizing radiation has been proven in the process of its exploitation, it can be recommended for use at other research beams of the IBR-2 reactor or any other similar nuclear-physics facilities or organizations operating with highly active radioactive wastes.

\section{REFERENCES}

1. M. Bulavin et al., "Irradiation facility at the IBR-2 reactor for investigating material radiation hardness," Nucl. Instrum. Methods Phys. Res., Sect. B 12 (2) (2014).

2. M. Bulavin et al., "Spectrum and density of neutron flux in the irradiation beam nine No. 3 of the IBR-2 reactor," Phys. Part. Nucl. Lett. 12, 336-343 (2015).

3. M. Bulavin and S. Kulikov, "Current experiments at the irradiation facility of the IBR-2 reactor," J. Phys.: Conf. Ser. 1021, 012041 (2018).

4. M. V. Bulavin and S. A. Kulikov, "Facility for radiation damage studies in materials and electronic compo- nents," Joint Inst. Nucl. Res. News, No. 1, 21-23 (2019).

5. M. V. Bulavin et al., "About model experiments on production of medical radionuclides at the IBR-2 reactor," in Proceedings of 26th International Seminar on Interaction of Neutrons with Nuclei. Fundamental Interactions, Neutrons, Nuclear Structure, Ultra Cold Neutrons, Xian, China, 2019, pp. 193-197.

6. V. Yu. Koval, A. Yu. Dmitriev, S. B. Borzakov, O. E. Chepurchenko, Yu. G. Filina, V. S. Smirnova, V. V. Lobachev, N. N. Chepurchenko, and M. V. Bulavin, "Ceramics of Bolgar: The first results of usage of neutron activation analysis," Phys. Part. Nucl. Lett. 16, 1004 (2019).

7. D. Chokheli et al., "Light yield and radiation hardness studies of scintillator strips with a filler," Nucl. Instrum. Methods Phys. Res., Sect. A 930, 87-94 (2019).

8. O. V. Ryabukhin et al., "Radiation coloration of the Ural topaz using electron and neutron radiation," in Proceedings of the 12th Issyk-Kul' International SchoolConference on Radiation Physics of Solid State SCORPh2017, Nauch. Zh. Fiz., No. 1, 55-57 (2017).

9. Yu. Khatchenko et al., "Study of neutron irradiationinduced color in topaz at the pulsed reactor IBR-2," in Proceedings of the 25th International Seminar on Interaction of Neutrons with Nuclei: Neutron Spectroscopy, Nuclear Structure, Dubna, May, 2017, pp. 22-26.

10. I. Bolshakova, "Experimental evaluation of stable long term operation of semiconductor magnetic sensors at ITER relevant environment," Nucl. Fusion 55, 083006 (2015).

11. I. Bolshakova et al., "Metal Hall sensors for the new generation fusion reactors of DEMO scale," Nucl. Fusion 57 (11) (2017).

12. M. V. Bulavin and S. A. Kulikov, "Robot to help you!," Dubna: Nauka Sodruzh. Progr., No. 48 (2018).

13. M. V. Bulavin and S. A. Kulikov, "Installation for investigation of radiation damage in materials and electronic components," Inform. Byull. Nov. OIYaI, No. 1 (2019).

14. SanPiN 2.6.1.2523-09, Radiation safety standards NRB-99/2009.

Translated by E. Smirnova 